\title{
Search for the effect of pressure on liquid-liquid critical phenomena
}

\author{
A KUMAR, V C VANI*, A G AIZPIRI ${ }^{\dagger}$ and R G RUBIO ${ }^{\dagger}$ \\ Department of Physics, Indian Institute of Science, Bangalore 560012, India \\ * Instrumentation and Services Unit, Indian Institute of Science, Bangalore 560012, India \\ ${ }^{\dagger}$ Departamento de Quimica Fisica, Facultad de Quimicas, Universidad Complutense, \\ 28040-Madrid, Spain
}

MS received 8 January 1992; revised 15 April 1992

\begin{abstract}
The coexistence curve of methanol + cyclohexane has been reanalyzed using an intrinsically simpler equation to study the influence of pressure on its asymmetry. The results confirm that no pressure dependence is discernible in the coexistence curve. Suggestions for future work in this direction are made.
\end{abstract}

Keywords. Liquid-liquid transitions; critical phenomena; pressure effect; critical exponents.

PACS Nos $64 \cdot 70 ; 64 \cdot 60$

\section{Introduction}

We intend to explore the effect of pressure (P) on liquid-liquid (L-L) critical phenomena (Kumar et al 1983; Sengers and Levelt Sengers 1986) employing the coexistence curve of a binary fluid mixture as an example. These systems belong to the Ising universality class (spin dimensionality, $n-1$ ). Pressure is not expected to affect (Kadanoff 1966; Griffiths 1970 and Stauffer et al 1972) the critical exponents and certain dimensionless amplitude ratios in binary fluid mixtures. This is a consequence of the fact that (a) $P$ is not a field conjugate to the order parameter (difference of concentration of one of the two fluids in both phases) and (b) $P$ does not change the symmetry of the order parameter.

At a highly quantitative level, the pressure independence of various universal parameters has been shown near the superfluid transition of liquid ${ }^{4} \mathrm{He}(n=2)$ (Ahlers 1980) and the Curie temperature of nickel (Yousuf and Kumar 1989) $(n=3)$. The $\lambda$-line in ${ }^{4} \mathrm{He}$ and the line of Curie temperatures in $\mathrm{Ni}$, do not display any pronounced curvatures when scanned with pressure. Historically the investigations along the $\lambda$-line of ${ }^{4} \mathrm{He}$ (Ahlers 1980) regarding the thermo-hydrodynamic quantities were pivotal in highlighting the significance of the confluent singularity (or correctionto-scaling) terms as well as in testing the results of the renormalization group theory (RGT) of phase transitions (Wilson and Kogut 1974; Le Guillou and Zinn-Justin 1980, 1989) in a rigorous manner.

The comprehension of multicritical points is better facilitated with the aid of pressure instead of an additional component - in binary (Prafulla et al 1992) and ternary (Schneider 1991) liquid mixtures.

A meticulous investigation concerning the influence of pressure on the L-L 
coexistence curve of methanol + cyclohexane $(\mathrm{M}+\mathrm{CH})$ has been reported (Aizpiri et al 1988). These data are carefully reanalysed using a simpler expression.

\section{Reanalysis of the data of Aizpiri et al}

Two of us (RGR and AGA) measured the coexistence curve (Aizpiri et al 1988) of $(\mathrm{M}+\mathrm{CH})$ in the pressure range $0 \cdot 1<\mathrm{P} / \mathrm{MPa}<13 \cdot 0$. Several isobars were obtained interpolating in various visually determined isopleths. The temperature, pressure and composition resolutions were $+1 \mathrm{mK}, \pm 0.01 \mathrm{MPa}$ and \pm 0.002 respectively. The shape of the phase diagram and its diameter (Greer 1976; Greer et al 1983; Vani et al 1986; Aizpiri et al 1988) can be combined to yield a general expression,

$$
\left|X-X_{c}\right|= \pm A_{1} t \pm A_{2} t^{1-\alpha}+A_{3} t^{\beta}+A_{4} t^{\beta+\Delta}
$$

where $t=\left|\left(T_{c}-T\right) / T_{c}\right|, T_{c}$ being the critical temperature. $X$ can be any concentration variable of the coexistence curve. $A_{1}-A_{4}$ are the parameters to be fitted. The critical exponents $\beta$ and a describe the shape of the coexistence curve and specific heat singularity respectively as $t \rightarrow 0$. According to RGT (Wilson and Kogut 1974; Le Guillou and Zinn- Justin 1980, 1989), $\beta=0.325$ and $a=0.110$. The correction-toscaling exponent (Greer 1978; Kumar et al 1983; Sengers and Levelt Sengers 1986) has a value $\mathrm{A}=0.50$ and $X_{c}$ is the critical concentration. When the chosen composition variable does not provide the preferred order parameter, the $t^{1-\alpha}$ term may be hidden under a strong $t^{2 \beta}$ anomaly, but very often the data do not allow to distinguish between the two (Greer 1978; Kumar et al 1983; Greer et al 1983). In the data analysis reported here, equation (1) is used to fit the data of Aizpiri et al (1988). The data were fitted using a nonlinear-least-squares fit program CURFIT (Bevington 1969). This program has been extensively used earlier for similar expressions (Greer 1976; Greer et al 1983; Vani et al 1986). The criterion for a good fit (Bevington 1969) was that

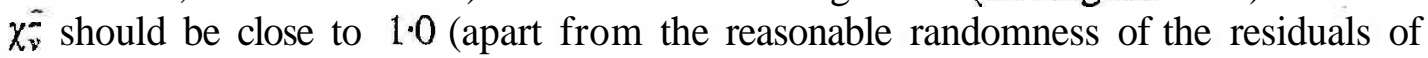
thefits).

The original data from Aizpiri et al (1988) i.e. $(T, P)$ at a given $X$, were converted to $(X, T)$ at a given $P$ by means of an interpolation method (Aizpiri et al 1988). These data were fitted to each branch of the coexistence curve to generate the coexisting compositions at a given $T$, from which the corresponding values of the order parameter and of the diameter were obtained and fitted. The essential conclusions (Aizpiri et al 1988) were that (a) P effect on the asymmetry of the coexistence curve, was masked by uncertainties and (b) the diameter shows no statistically significant anomaly. Here it should be noted that the original precision of the measurements, which was remarkable, possibly suffered due to two steps of interpolation (which could also introduce small systematic errors and as a consequence a certain systematicity in the distribution of residuals). Since this research was the first careful endeavour to probe the pressure effect on $\mathrm{L}-\mathrm{L}$ critical phenomena, it was decided to reanalyze and reexamine these data using (1). The temperature range covered in the data analysis is $1.5 \times 10^{-4} \leqslant t \leqslant 5 \times 10^{-2}$ which is outside the range of gravity effects for $(\mathrm{M}+\mathrm{CH}$; even for the smallest $t$ approached (Kumar et al 1983; Greer 1978; Aizpiri et al 1988). The two data points closest to $T_{\boldsymbol{c}}$ were neglected as was done in the reference of Aizpiri et al (1988). The critical exponents a, $f t$ and A were kept fixed at their theoretical values as is the common practice (Greer et al 1983; Aizpiri et al 1988). 
The minimum in $\chi_{v}^{2}$ was achieved by varying $X_{c}$ and $T_{c}$. These values of $X_{c}$ and $T_{c}$ were then held fixed and parameters $A_{1}-A_{4}$ were determined. The term $A_{4} t^{\beta+\Delta}$ in (1) was not found to improve the fit significantly and was discarded. This result is in agreement with the assumption that for L-L critical systems the first correctionto-scaling amplitude is small as has been reported recently (Singh and Pitzer 1989).

It is also inferred that the anomalous term, $A_{2} t^{1-\alpha}$, in the diameter [equation (1)] cannot be excluded from the data - this finding is not in consonance with the work of Aizpiri et al (1988). However, a careful examination of the residuals of the diameter fits of Aizpiri et al (1988) (unpublished) does not rule out the possibility that those data were consistent with the existence of a $(1-\mathrm{a})$ term in (1).

According to our results, amplitude $A_{2}$ seems to be the best index to reveal the effect of $P$ on the coexistence curve of $(\mathrm{M}+\mathrm{CH})$. Equation (1) treats the order parameter as mole fraction, which is the preferred order parameter for this system (Greer et al 1983; Aizpiri et al 1988). The results of the fit are shown in tables 1 and 2.

Table 1. Results of fits to equation (1) [Right: $R\left(X>X_{c}\right)$ Left: $\mathrm{L}\left(X<X_{c}\right)$ ]. The values of $X_{c}$ and $T_{c}$ were kept fixed. A small variation in steps of \pm 0.001 for $X_{c}$ did not affect the results. The right branch of the coexistence curve gave a better fit than the left branch, possibly due to paucity of data points very close to $X_{\mathrm{c}}$.

\begin{tabular}{|c|c|c|c|c|c|}
\hline $\mathrm{P} / \mathrm{MPa}$ & $T_{c} / \mathrm{K}$ & $A_{1}$ & $A_{2}$ & $A_{3}$ & $\chi_{v}^{2}$ \\
\hline $0.1 \mathrm{R}$ & 318.445 & $-11 \cdot 1 \pm 0.8$ & $8.1 \pm 0.6$ & $0.79 \pm 0.01$ & 1.02 \\
\hline $0.1 \mathrm{~L}$ & 318.445 & $14 \cdot 2 \pm 1 \cdot 1$ & $-11.6 \pm 0.9$ & $0.69 \pm 0.02$ & 0.07 \\
\hline $1 \mathrm{R}$ & 318.749 & $-11.9 \pm 0.8$ & $8.7 \pm 0.6$ & $0.79 \pm 0.01$ & 0.96 \\
\hline $1 \mathrm{~L}$ & $318 \cdot 749$ & $9.9 \pm 1.0$ & $-8.4 \pm 0.8$ & $0.74 \pm 0.01$ & 0.21 \\
\hline $2 \cdot 5 \mathrm{R}$ & $319 \cdot 264$ & $-11.2 \pm 0.8$ & $8.2 \pm 0.6$ & $0.79 \pm 0.01$ & $1 \cdot 18$ \\
\hline $2 \cdot 5 \mathrm{~L}$ & $319 \cdot 264$ & $15 \cdot 3 \pm 1 \cdot 2$ & $-12.6 \pm 0.9$ & $0.67 \pm 0.02$ & 0.02 \\
\hline $5 \mathrm{R}$ & 320.070 & $-11.8 \pm 0.8$ & $8.6 \pm 0.6$ & $0.79 \pm 0.01$ & 1.06 \\
\hline $5 \mathrm{~L}$ & 320.070 & $10.5 \pm 0.9$ & $-8-7 \pm 0 \cdot 8$ & $0-75 \pm 0 \cdot 01$ & 0.70 \\
\hline $10 \mathrm{R}$ & 321.688 & $-11.5 \pm 0.8$ & $8 \cdot 6 \pm 0.6$ & $0.77 \pm 0.01$ & 0.51 \\
\hline $10 \mathrm{~L}$ & 321.688 & $14 \cdot 4 \pm 1 \cdot 1$ & $-11.9 \pm 0.9$ & $0.68 \pm 0.02$ & 0.10 \\
\hline $13 \mathrm{R}$ & $322 \cdot 600$ & $-9-9 \pm 0.7$ & $7.2 \pm 0.6$ & $0.80 \pm 0.01$ & $1 \cdot 13$ \\
\hline $13 \mathrm{~L}$ & $322 \cdot 600$ & $15-5 \pm 1 \cdot 0$ & $-12.4 \pm 0.8$ & $0.69 \pm 0.02$ & 0.17 \\
\hline
\end{tabular}

Table 2. Ratios of the amplitudes $A_{1}, A_{2}$ and $A_{3}$ for the right and left branches (taken from table 1). These ratios are expected to articulate the effect of pressure on the coexistence curve. None of the ratios show any systematic trend up to $13 \mathrm{MPa}$.

\begin{tabular}{|c|c|c|c|c|}
\hline & & $A_{1}(\mathrm{R})$ & $A_{2}(\mathrm{R})$ & $A_{3}(\mathrm{R})$ \\
\hline $\mathrm{P} / \mathrm{MPa}$ & $T_{e} / \mathrm{K}$ & $A_{1}(\mathrm{~L})$ & $A_{2}(\mathrm{~L})$ & $A_{\mathbf{3}}(\mathrm{L})$ \\
\hline 0.1 & $318 \cdot 445$ & $-0-783$ & -0.701 & $1 \cdot 141$ \\
\hline 1 & 318.749 & -1.203 & -1.039 & 1.070 \\
\hline 2.5 & $319 \cdot 264$ & -0.732 & -0.652 & $1 \cdot 186$ \\
\hline 5 & 320.070 & $-1 \cdot 120$ & -0.984 & 1.061 \\
\hline 10 & 321.688 & -0.800 & -0.720 & 1.136 \\
\hline 13 & 322.600 & $-0-642$ & -0.579 & $1 \cdot 158$ \\
\hline
\end{tabular}




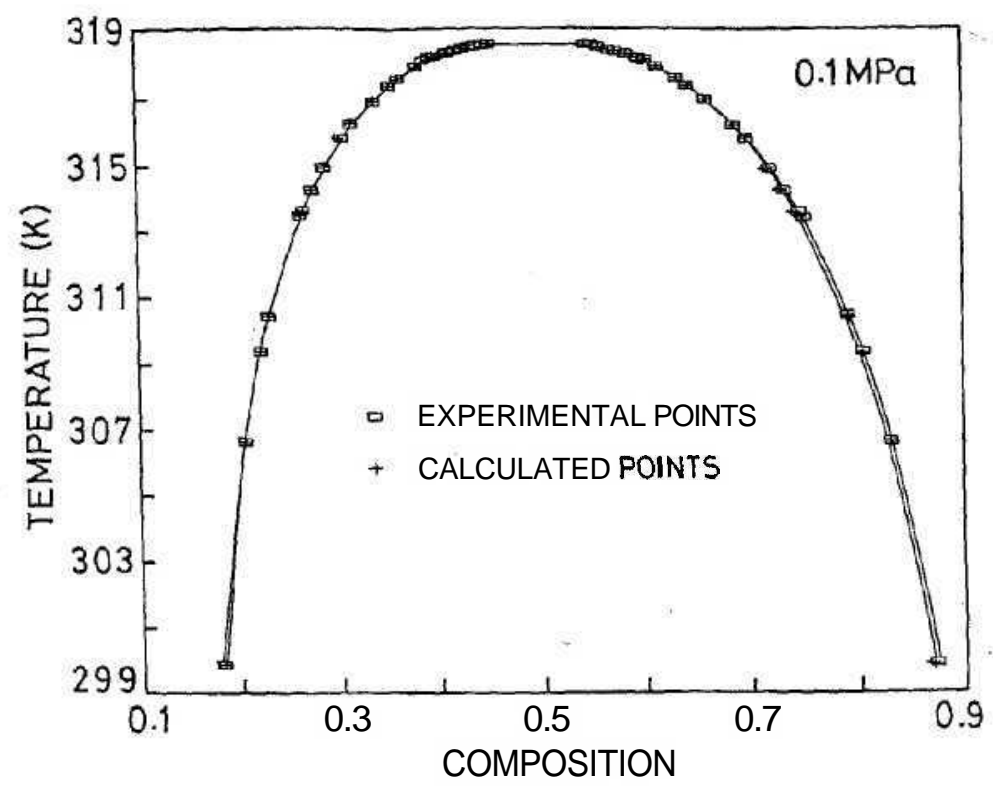

Figure I. Experimental and calculated phase diagram (using equation (1)) of methanol+ cyclohexane at $0.1 \mathrm{MPa}$. The continuous line is guide to the eye.

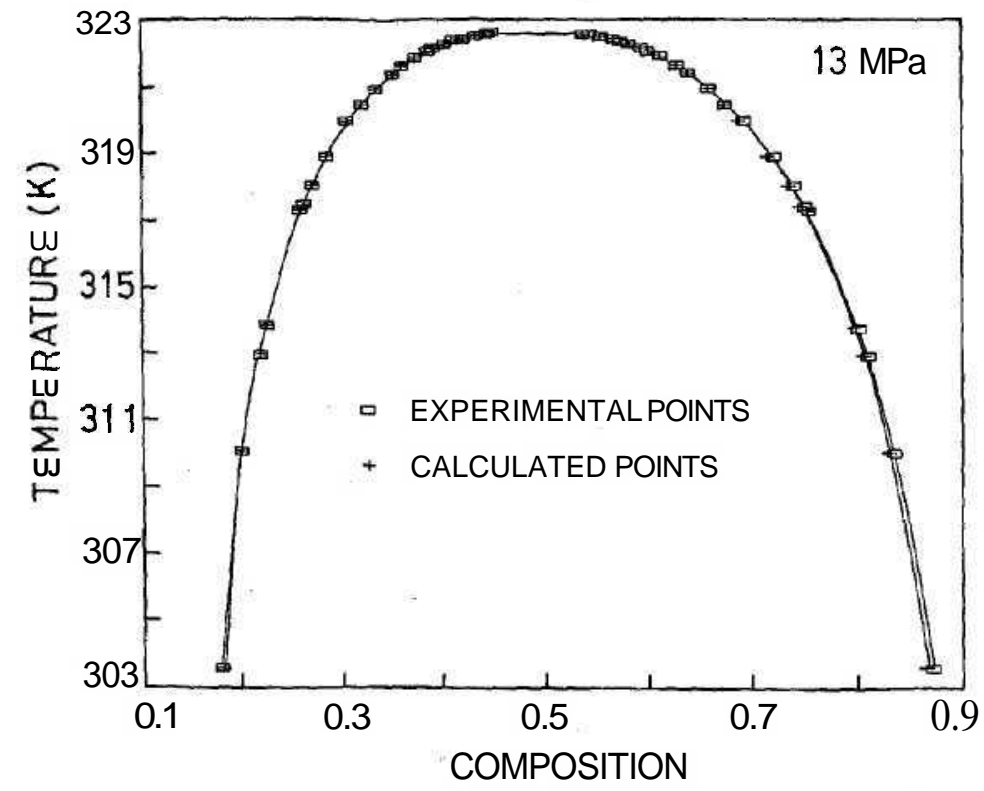

Figure 2. Experimental and calculated phase diagram (using equation (1)) of methanol + cyclohexane at $13 \mathrm{MPa}$. The continuous line is guide to the eye.

Figures 1 and 2 display the complete coexistence curve at two extreme pressures along with the fitted values.

\section{Discussion}

It is evident from tables -1 and 2 that there is no noticeable effect of $P$ on $A_{2}$ i.e. the increased pressure has not enhanced the asymmetry of the coexistence curve. A similar result persists if $(1-a)$ is replaced by $2 \beta$ in (1). The non observation of 
the effect of $P$ is not in conflict with the ideas of universality and the RGT (Kadanoff 1966; Griffiths 1970; Stauffer et al 1972; Wilson and Kogut 1974; Le Guillou and Zinn-Justin 1980, 1989).

The debatable question is whether the effect of pressure on the $\mathrm{L}-\mathrm{L}$ coexistence curve can be measured at all. Here one must refer to the situation in the study of the superfluid transition in liquid ${ }^{4} \mathrm{He}$ as pressure was increased to nearly $3 \mathrm{MPa}$ along the $\lambda$-line (Ahlers 1980). The pressure dramatically increased the amplitude of correction-to-scaling terms for the superfluid fraction, thermal expansion and thermal conductivity. Nonetheless, it should be recognized that a modest pressure of $\simeq 3 \mathrm{MPa}$ is fairly close to the freezing pressure for liquid ${ }^{4} \mathrm{He}$. Perhaps one has to extend the range of pressure to a few hundred MPa in binary fluid systems to observe its influence. The ultimate pressure will be limited by the solidification of one of the liquids.

\section{Suggestions for future work}

It is fortuitous that a highly symmetric system (i.e. a system with $X_{c}$ almost at 0.5 and a very symmetric coexistence curve), $(\mathrm{M}+\mathrm{CH})$, was chosen to see the effect of pressure. It seems prudent to choose a system like polystyrene + cyclohexane, that is extremely asymmetric, for a molecular weight of $10^{5}$ or $10^{6}$ of polystyrene. The critical volume fraction of polystyrene (Nakata et al 1975, 1978) is 0.0321 in this system.

It is suggested that a direct isobaric determination of the coexistence curve should be made, preferably with a single sample technique, avoiding interpolation at any step.

\section{Acknowledgements}

We have benefited from our discussions with E S R Gopal. We are indebted to $\mathrm{T}$ Narayanan for his suggestions. We thank B V Prafulla for a thorough reading of the manuscript. The financial support to AK and VCV from the Department of Atomic Energy and Indo-French Centre for the Promotion of Advanced Research is gratefully acknowledged. AGA and RGR received partial support for this work from the Universidad Complutense under a grant for Grupos Precompetitivos.

\section{References}

Ahlers G 1980 Rev. Mod. Phys. 52489 and references therein

Aizpiri A G, Rubio R G and Pena M D $1988 \mathrm{~J}$. Chem. Phys. 881934 and references therein Bevington P R 1969 Data reduction and error analysis for the physical sciences (New York: McGraw Hill)

Greer S C 1976 Phys. Rev. A14 1770

Greer S C 1978 Acc. Chem. Res. 11427

Greer S C, Das B K, Kumar A and Gopal E S R 1983 J. Chem. Phys. 794545

Griffiths R B 1970 Phys. Rev. Lett. 241479

Kadanoff L P 1966 Physics (New York) 2263

Kumar A, Krishnamurthy H R and Gopal E S R 1983 Phys. Rep. 9857

Le Guillou J C and Zinn-Justin J 1980 Phys. Rev. B21 3976

Le Guillou J C and Zinn-Justin J 1989 J. Phys. (Paris) 501365

Nakata M, Dobashi T, Kuwahara N and Kaneko M 1978 Phys. Rev. A18 2683 
Nakata M, Kuwahara N and Kaneko M 1975 J. Chem. Phys. 624278

Prafulla B V, Narayanan T and Kumar A 1992 Phys. Rev. A45 1266

Schneider G M 1991 J. Chem. Thermodyn. 23301 and the literature cited

Sengers J V and Levelt-Sengers J M H 1986 Ann. Rev. Phys. Chem. 37189

Singh R R and Pitzer K S 1989 J. Chem. Phys. 905742

Stauffer D, Ferer M and Words M 1972 Phys. Rev. Lett. 29345

Vani V, Guha S and Gopal E S R 1986 J. Chem. Phys. 843999

Wilson K G and Kogut J 1974 Phys. Rep. C12 75

Yousuf M and Kumar A 1989 Phys. Rev. B39 7288 